\title{
Investigation of the Effectuation of Graphene Nanosheets (GNS) Addition on the Mechanical Properties and Microstructure of S390 HSS Using Powder Metallurgy Method
}

\author{
H. M. Zidan' ${ }^{1}$, M. Hegazy ${ }^{2}$, A. Abd-Elwahed ${ }^{2}$, H. M. Yehia ${ }^{3}$, O. A. El-Kady ${ }^{1, *}$ \\ ${ }^{1}$ Powder Technology Division, Central Metallurgical R\&D Institute (CMRDI), Egypt. \\ ${ }^{2}$ Dept. of Physical Chemistry, Faculty of science, Helwan University, Egypt. \\ ${ }^{3}$ Dept. of production Technology, Faculty of Technology and Education, Helwan University, Egypt.
}

*Corresponding author e-mail address: o.alkady68@gmail.com

Received 23 March 2021

Revised 26 May 2021

Accepted for publication 27 May 2021

Published online 30 June 2021

\section{Abstract}

The effect of graphene-nanosheets (GNs) on the mechanical properties and microstructure of the S390 HSS alloy was investigated. The S390 HSS sample reinforced with $0.25,0.5$, and $0.75 \mathrm{wt}$.\% GNS. Powder metallurgy technique was used to prepare all samples. The elemental powders of the alloy were milled in a planetary ball mill by 10:1 ball to powder ratio with speed $350 \mathrm{rpm}$ for 48 hours, and the mixed powders were warm compacted by the uni-axial press at $550^{\circ} \mathrm{C}$ under $500 \mathrm{MPa}$ and then sintered in a vacuum furnace at $1370^{\circ} \mathrm{C}$ for 1 hour. X-ray diffraction shows the formation of secondary carbide phases and martensite. The density result indicates the decreasing of density values by GNS additions. The mechanical properties show an increase in the hardness and wear resistance by increasing the graphene content. The specimens are metallographically examining using Scanning Electron Microscopy (SEM), and also the electron backscatter diffraction shows a good distribution of GNS in the HSS matrix.

Keywords: High-speed steel; Nano-graphene sheets; Powder metallurgy; Mechanical milling; Microstructure

\section{Introduction}

High-speed steel is one of the most important cutting tool materials that must continuously improve and develop to be with the industrial world progress. It belongs to the FeCX high alloy steel; $\mathrm{X}$ is the challenging carbide-forming element like chromium, tungsten, molybdenum, and vanadium [1]. Graphene is a cermaic material and considered the best choice as reinforcement to improve the mechanical properties of high-speed steel alloy [2]. It has unique properties that make it harder in structure than diamond and has high flexibility that makes it quickly cover the surface of many materials in different forms..

By the traditional production methods of HSS such as melting and casting, the graphene can't mix homogeneously with the ingot because of its low density and high melting point it will float on the sample's surface and also when an alloy with a high percentage of carbon undergoes slow cooling of ingot in casting process lead to a formation of coarse as-cast microstructure composed of coarse eutectic W, Mo and $\mathrm{V}$ rich carbide which decrease the toughness and flexibility of the HSS tool. So for good mixing of graphene with the alloy content and to prevent the formation of coarse eutectic carbides, the powder metallurgy technique has been used as a manufacturing process [3].

Powder metallurgy route is considered a shortprocess manufacturing method used to produce refined steel free from any inclusions and has a uniform microstructure. These properties can't be obtained by the conventional casting process [4-6].

Many investigations were established on using the P/M technique for high-speed steel processing. Zhong improved the toughness, wear-resistance and get uniform microstructure to the high-speed steels AHPT1 $\left(\mathrm{W}_{12} \mathrm{Cr}_{4} \mathrm{~V}_{5} \mathrm{Co}_{5}\right)$ by gas atomization (one of powder metallurgy method), hot isostatic pressing, hot working, and heat treatment processes [7].

MatjažGodec studied the microstructural characterization of the powder-metallurgy high-speedsteel S390,a uniform distribution of vanadium and tungsten carbides in the steel matrix [8]. Abdul Manaf used the powder metallurgy technique to improve the hardness of M3/2 HSS by mixing it with (Fe3P) powders in a tubular mixer for half an hour by dry 
mixing [9]. D. Zhang et al.was felicitous in formed HSS by solid-state sintering of $\mathrm{Fe}, \mathrm{Co}, \mathrm{WC}, \mathrm{Mo}_{2} \mathrm{C}, \mathrm{Cr}_{3} \mathrm{C}_{2}$, and $\mathrm{VC}$ powders in a vacuum furnace then a heat treatment of the sintered samples was done, which reveals that the microstructure investigation shows that the base matrix is a martensitic structure with asymmetrical distribution of MC and M6C carbides [10]. From the above investigations it can be conclude that powder technology is an effective rout in the manufacturing and improving the mechanical properties of HSS.

Mechanically alloying is one of powder metallurgy methods, which consider a very successful protocol for forming materials with a well-knit chemical composition and microstructural features that were difficult to be obtained using other synthesis routes [11]. In this work, the mechanical properties of the S390 MC high-speed steel are improved by adding grapheme-nanosheets using the mechanical alloying method to enhance the sintered samples' hardness and tribological properties.

\section{Experimental work}

High-speed steel PM S390 MC is manufactured from commercially available metal powders $(\mathrm{Fe}, \mathrm{V}, \mathrm{Co}, \mathrm{Cr}$, $\mathrm{C}, \mathrm{W}$, and Mo). The chemical composition of the alloy is given in Table (1) according to BÖH LER S390 MICROCLEAN alloy.

Table 1. The chemical composition of $\mathrm{S} 390$ Microclean high speed steel alloy.

\begin{tabular}{|c|c|c|c|c|c|c|}
\hline Elements & $\mathrm{C}$ & $\mathrm{Cr}$ & $\mathrm{Mo}$ & $\mathrm{V}$ & $\mathrm{W}$ & $\mathrm{Co}$ \\
\hline $\begin{array}{c}\text { wt\% balanced } \\
\text { with Fe }\end{array}$ & 1.6 & 4.8 & 2 & 5 & 10.5 & 8 \\
\hline
\end{tabular}

The properties of each element are illustrated in Table (2).

Table 2. The particle size and the purity of the alloying elements.

\begin{tabular}{|c|c|c|}
\hline Iron $(\mathrm{Fe})$ & $10 \mu \mathrm{m}$ particle size. & $99.98 \%$ \\
\hline Carbon(C) & $5 \mu \mathrm{m}$ particle size & $99.8 \%$ \\
\hline Chromium (Cr) & 100 mesh particle size & $99.9 \%$ \\
\hline Molybdmenum (Mo) & 100 mesh particle size & $99.9 \%$ \\
\hline Vanandium (V) & in shape of pellets & $99.5 \%$ \\
\hline Tungsten (W) & 50 mesh particle size. & $99.7 \%$ \\
\hline Cobalt (Co) & $2 \mu \mathrm{m}$ particle size. & $99.95 \%$ \\
\hline
\end{tabular}

Different percent added graphene-nanosheets of 2-10 nm thickness supplied by ACS Material, LLC (Advanced Chemical Supplier) (0, 0.25, 0.5, and 0.75 wt. \%). Graphene is a ceramic material, and its wettability between it and metals powders is weak, so graphene surface must be metalized firstly, by surface coating with silver metal, by using the silver chemical bath which contains: silver nitrate of $99.9 \%$ purity, ammonia $33 \%$, and formaldehyde of $38 \%$ concentration. 2 wt. $\%$ of silver precipitate on the surface of graphene powder by dissolved silver nitrate in deionized water, then adding $33 \%$ ammonia solution to adjust $\mathrm{PH}$ at 11 , finally adding formaldehyde to start the reaction. After that, the metalized graphene powder was filtered, and then dried in a dryer at $80^{\circ} \mathrm{C}$ for 1 hour [12]. All chemicals supplied from El-gomhoria Company for chemicals - Cairo- Egypt. After the metallization process of graphene, it was mix with the elementary powders in a planetary ball mill with 350 rpm speed and 48 hour milling time. The addition of $1.5 \mathrm{wt} \%$ hexane as a process controlling agent (PCA) is added to the powder mixture to decrease the cold welding effect during the milling process. The ball to powder ratio is $10: 1$, and the process occurred under argon gas to protect the metal powders from any oxidation. The mixed powders are warm pressed at $550^{\circ} \mathrm{C}$ using auni-axial press in a $\mathrm{K} 110$ tool steel circular die with a diameter of $12 \mathrm{~mm}$ and pressed under a $500 \mathrm{MPa}$,finally sintered in a vacuum furnace at $1370{ }^{\circ} \mathrm{C}$ by a heating cycle shown in Fig. 1 . There is one holding step at $800^{\circ} \mathrm{C}$ for $30 \mathrm{~min}$ for cobalt liquefaction and another holding time one hour at 1370 ${ }^{\circ} \mathrm{C}$ to complete sintering process.

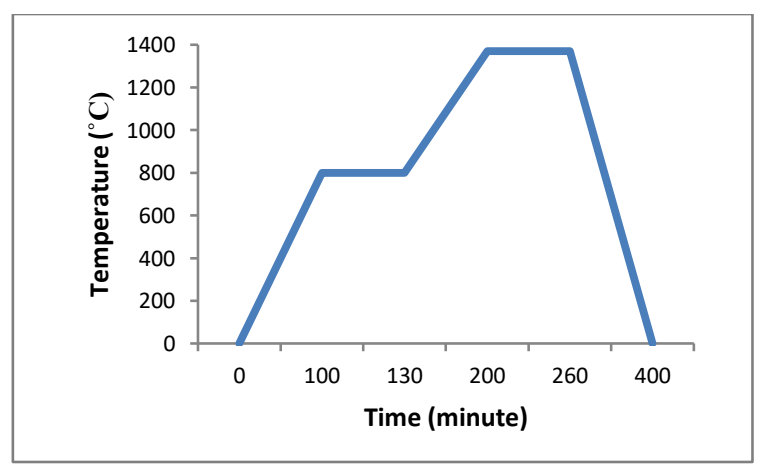

Fig. 1 The sintering heat cycle in the vacuum furnace.

\section{Characterization}

\subsection{Density measurements}

The density of samples after sintering is calculated using the Archimedes method. The distilled water is used as a floating liquid according to MPIF standards 42, 1998, and the sintered materials are weighed in air and then in distilled water. The density of samples was calculated according to the following equation $(\mathrm{Eq}(1))$.

$\operatorname{Density}\left(\mathrm{gm} / \mathrm{cm}^{3}\right)=$

[Wt. in air / (Wt. in air - Wt. in water)] 


\subsection{Phase and microstructure identification}

The phase identification of the milled powder and the sintered samples were investigated by $\mathrm{x}$-ray diffraction analysis (XRD) using $\mathrm{x}$-ray diffractmeter model $\mathrm{x}$, pert PRO PANalytical with $\mathrm{Cu} k \alpha$ radiation $(\lambda=0.15406$ $\mathrm{nm})$. For microstructure investigation, he surfaces of the sintered samples were prepared by grinding and polishing using silicon carbide papers with different grit (400, 600, 800, 1000, and2000respectively) and finally with diamond paste. The metallographic structure of the sintered specimens were examined by scan electron microscope model TESCAN SEM VEGA3.

\subsection{Mechanical properties}

\subsubsection{Hardness test}

Vickers hardness (HV) is measured as the average of 5 readings along the polished cross-section surface of the specimen using Vickers hardness tester model InstronWolpert type $930 / 250$ by applying $10 \mathrm{Kg}$ load and $15 \mathrm{sec}$ load time.

\subsubsection{Tribological test (The friction coefficient and wear rate test)}

The tribological test was applied on the prepared sample using a (pin-on-disk) tester machine that works according to the SAE-J661 standard test [13]. As shown in Fig. 2, the machine consists of a typical automobile brake disc (made from a grey cast iron alloy) used as a frictional disk. The machine has an instrumental diagnosticsystem used for disc rotation speed and specimen friction force detection. Finally, the friction system noise has been detected by a 2240 (SLM) sound level meter.

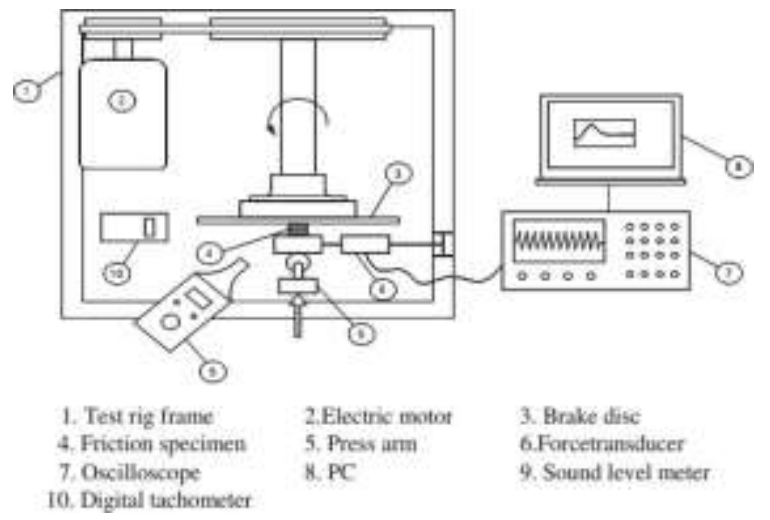

Fig. 2 Arrangement of the pin-on-disc tester and its diagnostic instrumentation system.

\subsection{2.a Coefficient of friction}

The friction coefficient of the samples is detected according to the following parameters $50 \mathrm{~N}$ friction force and $7 \mathrm{~m} / \mathrm{s}$ sliding speed of the disc, that's because the friction of materials depends on the testing time, loading force, and sliding speed, which is performed.

\subsection{2.b Wear rate}

The wear rate was detected by using the same (pinon-disk) machine. The wear rate depends on the sample's weight loss with time.Itwas measured according to the following parameters $(50 \mathrm{~N}$ applied force on the sample and $18 \mathrm{~min}$ time for each sample).

\section{Results and discussion}

\subsection{Density measurement}

Fig. 3 shows the relative change in the density of the sintered samples. It was found that as the percentage of graphene increases, the sample's density decreases. That decrease occurred due to the lower density value of the graphene nanosheets $(2.2 \mathrm{~g} / \mathrm{cm} 3)$ that is lower than the density of the other alloy elements. So by the addition of a light element to the alloy cause a decrease in the final composite density [14].

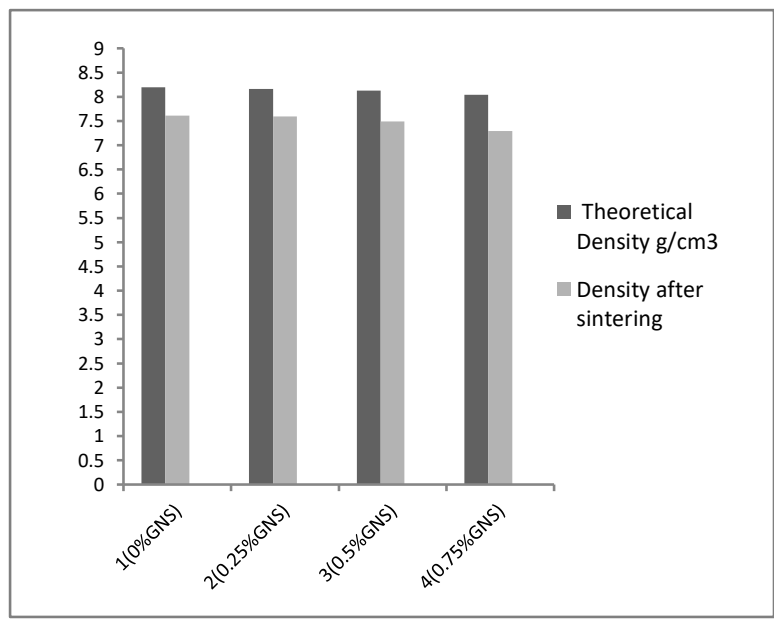

Fig. 3 The effect of GNS on the theoretical and measured density.

\subsection{X-ray diffraction phase structure and composition}

Fig. 4 shows the XRD pattern of S390 HSS powder after the mechanically milling process that reveals the presence of $(\gamma)$ iron (Austenite) and also a tiny peak of $(\alpha)$ iron (Martensite). Four principal carbide phases were identified, and the most significant peak corresponds to $\mathrm{Fe}_{3} \mathrm{~W}_{3} \mathrm{C}$ and the smallest one for $\mathrm{Cr}_{2} \mathrm{C}_{3}$. The other two peaks for $\mathrm{VC}$ and $\mathrm{M}_{6} \mathrm{C}$ (Tungsten-rich carbides) [15] and finally a peak for molybdenum metal at $2 \theta 74$ degrees. 


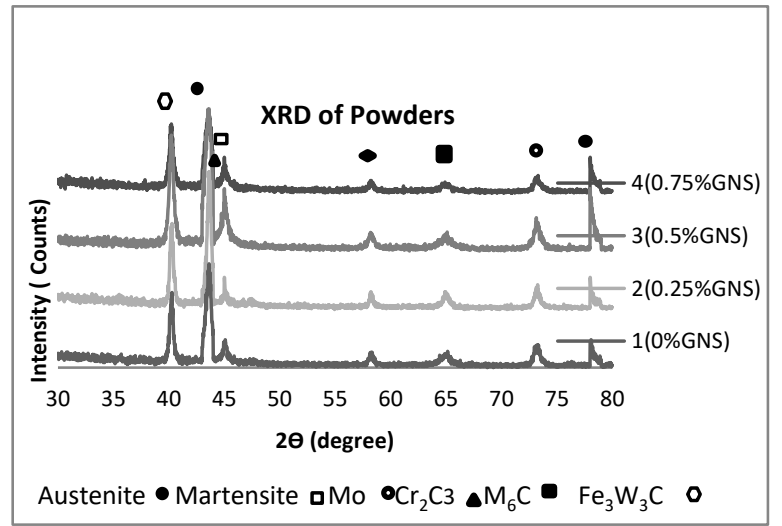

Fig. 4 XRD pattern of the powder samples after milling.

Fig. 5 shows the XRD pattern of S390 HSS reinforced with GNS samples after the sintering process. Three peaks belong to the $(\alpha)$ iron (Martensitic) phase, while $(\gamma)$ iron (Austenite) was disappeared. Also, there are no peaks for the $\mathrm{Fe}_{3} \mathrm{~W}_{3} \mathrm{C}$ phase, which means the dissolving of this carbide in the steel matrix during sintering [16] while the other carbide peaks, $\mathrm{VC}$ and $\mathrm{Cr}_{2} \mathrm{C}_{3} \mathrm{M}_{6} \mathrm{C}$ are recorded and didn't change.

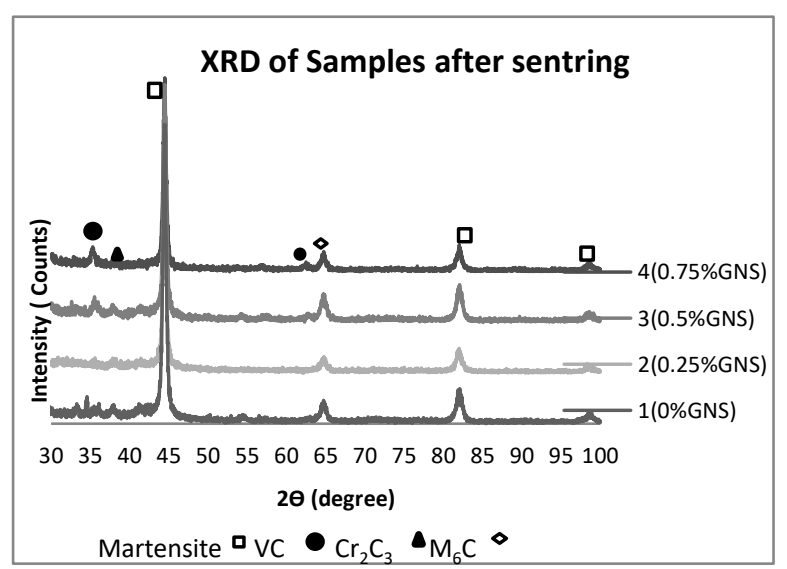

Fig. 5 The XRD pattern of samples after sintering.

\subsection{Microstructure analysis}

Fig. 6 shows the microstructure of the prepared samples. There are three areas observed: the gray area representing the alloy matrix that considers the martensitic phase. The second is the long white regions that belong to the formed primary eutectic carbides $\left(\mathrm{M}_{2} \mathrm{C}\right)(\mathrm{Mo}$ and $\mathrm{W}$ rich regions) [17]. In the black region, some formed secondary carbides (VC). The microstructure refers to the good distribution of the primary and secondary carbides all over the alloy matrix.Also, increasing the interaction between all the constituents indicatea good sintering process. For the high GNS percent, coarse particles are observed, in which some aggregations of the formed carbides occur.

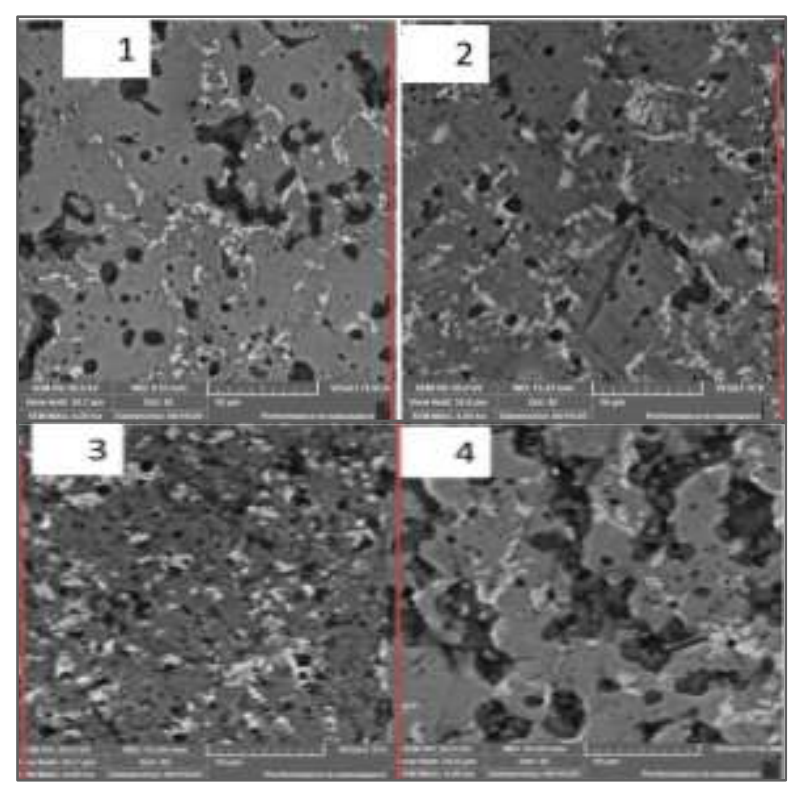

Fig. 6 The microstructure of the sintered samples.

\subsection{Hardness measurement}

Fig. 7 shows the HVvalues that consider an average of 5 readings. It shows a gradual increase in the hardness values of HSS by increasing the GNS content. Graphenehas a thickness layer with a hexagonal atomic structure.

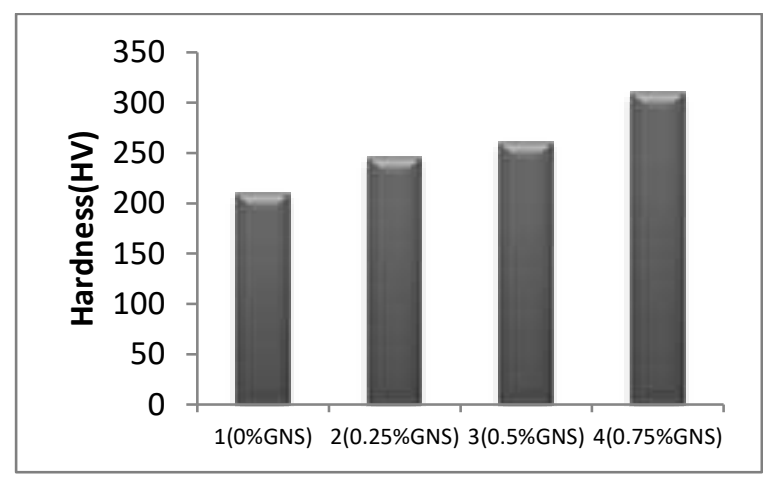

Fig. 7 The hardness after sintering.

This atomic structure gives the graphene high bonding strength than that found between the rest constituents of the alloy. It considers the most robust material that has been ever tested with a $130.5 \mathrm{GPa}$ tensile strength and $1 \mathrm{TPa}$ [18]. The graphene in the steel matrix works as a network, which holds the particles of the steel matrix and increases its strength, and finally leads to an increase in hardness [19]. Coating the graphene with silver leads to an improvement in the wettability and the adhesion with the constitutes of the steel. Also, the excellent distribution of the graphene all over the alloy matrix gives a good strength for the prepared alloy. 


\subsection{Wear and friction coefficient}

\subsubsection{Wear test}

Fig. 8 shows the weight loss for the sintered samples by grams with the graphene percent. The wear resistance increases as the graphene percent increases due to the formation of primary carbides by large amount during the solidification process, which gives a high strength to the HSS alloy. The addition of grapheme-nanosheets decreases the wear rate due to the lubricant nature of GNS [20]. Also, the coating GNS with $\mathrm{Ag}$ enhances the interconnection between all the constituents, decreasing the wear weight loss.

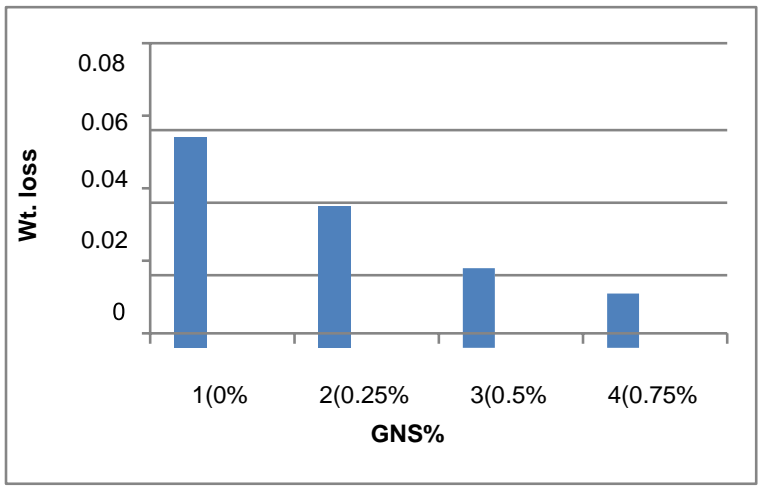

Fig. 8 Effect of GNS on the weight loss of the sintered samples.

\subsubsection{Coefficient of friction}

The variation in the friction coefficient with time in Fig. 9 shows that the friction coefficient increases by increasing the graphene percent because graphene is a phase of carbon.

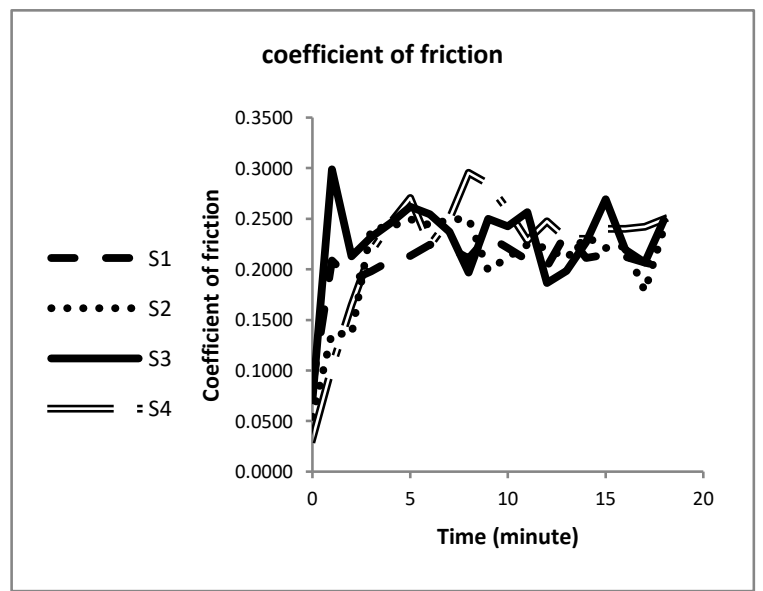

Fig. 9 Coefficient friction of samples.

Milling and sintering give more opportunity to form more carbides like $\mathrm{Cr}_{2} \mathrm{C}_{3}$ and $\mathrm{VC}$.

\section{Conclusions}

Four samples with different percent of GNS $(0,0.25$, 0.5 , and 0.75 ) have formed by the powder metallurgy method. The microstructure, density, hardness, tribological properties were investigated. The results showed well-distributed carbides through the steel matrix and although excellent reinforcement adhesion. It was found the density of the sintered samples decrease by increasing the GNS percentage. That due to the low density of graphene with regards to the other alloy content. Unlike density, there is a gradual increase in the hardness by increased the GNs percentage gradually. Also, the wear resistance increases gradually as the percent of GNS increases due to increased carbide content in the steel matrix. Finally, there is an unusual observation in the fraction coefficient as it increases by increasing the graphene percent, and that's because of the milling process, which makes graphene (as it is a phase of carbon) form more carbides like $\mathrm{Cr}_{2} \mathrm{C}_{3}$ and $\mathrm{VC}$, which hard and causes increases in the fraction coefficient.

\section{Acknowledgement}

First of all, I would like to express my deepest thanks for the almighty ALLAH. I want to present my thanks and gratitude to my coworker Eng. Ahmed Osama Abd el-Aleem and all technicians of powder metallurgy lap for their continued support and encouragement through working in this research. The authors also want to thank the Central Metallurgical R\&D Institute (CMRDI) in Helwan, Cairo, Egypt, for their support.

\section{References}

[1] H. Tang, H. Zhang, L. Chen, S. Guo, Novel laser rapidly solidified medium- entropy high speed steel coatings with enhanced hot wear resistance, Journal of Alloys and Compounds 772 (2019) 719 $-729$. https://doi.org/10.1016/j.jallcom.2018.09.122

[2] Ö. Güler, N. Bagc1, a short review on mechanical properties of graphene reinforced metal matrix composites, J. Mater. Res. and Techn. 9 (2020) 6808-6833. https://doi.org/10.1016/j.jmrt.2020.01.077

[3] C.Tornberg and A. Fölzer, Newoptimized manufacturing route for PM tool steels and high speed steels, Böhler-Uddeholm Powder Technology, 6th International Tooling Conference: 363-376.

[4] M. Hossam,A. Yehia, A. El-Tantawy, I.M. Ghayadb, A. S. Eldesoky, O. El-kady, Effect of zirconia content and sintering temperature on the density, microstructure, corrosion, and 
biocompatibility of the Ti-12Mo matrix for dental applications, J. of mater. Research and Tech. 9 (2 $020)$ 8820-8833. https://doi.org/10.1016/j.jmrt.2020.05.109

[5] M. A. Hassan, H. M. Yehia, A. S. A. Mohamed, A. E. El-Nikhaily, O. A. Elkady, Effect of Copper Addition on the AlCoCrFeNi High Entropy Alloys Properties via the Electroless Plating and Powder Metallurgy Technique, Crystals 11(5) (2021) 540. https://doi.org/10.3390/cryst11050540

[6] H. M.Yehia, S. Allam, S. Hot Pressing of Al-10 wt $\% \quad \mathrm{Cu}-10 \quad$ wt $\% \quad \mathrm{Ni} / \mathrm{x} \quad\left(\mathrm{Al}_{2} \mathrm{O}_{3}-\mathrm{Ag}\right)$ Nanocomposites at Different Heating Temperatures. Met. Mater. Int. 27 (2021) 500513.

https://doi.org/10.1007/s12540-020-00824-4

[7] H. Zhong, Y. Fang, C. Kuang, X. Kuang, Q. Hao, X. Li, Development of powder metallurgy high speed steel, Materials Science Forum 638-642 (2010) 1854- 1859.

https://doi.org/10.4028/www.scientific.net/MSF.6 $\underline{38-642.1854}$

[8] M. Godec, B. ŠetinaBatič, D. Mandrinoa, A. Nagode, V. Leskovšek, S. D. Škapin, M. Jenko, Characterization of the carbides and the martensite phase in powder-metallurgy high-speed steel, Materials Characterization 61(2010) 452 - 458. https://doi.org/10.1016/j.matchar.2010.02.003

[9] S. Abdul Manaf, A. A. Mahaidin, M. A. Selamat and T. R. Jaafar, A study on sintering behavior and mechanical properties of high speed steel powder through powder metallurgy route, Solid State Science and Technology 19 (2011) 170-183.

[10]Dan Zhang, Zhi Li, Lang Xie , Yifeng Xiao , FuchengYina, Powder metallurgy of high speedsteel produced by solid state sintering and heat treatment, International Journal of Materials Research 106 (2015) 870-876. https://doi.org/10.3139/146.111239

[11]J. M. Torralba , L. Fuentes-Pacheco , N. GarcíaRodríguez, M. Campos, Development of high performance powder metallurgy steels by highenergy milling, Adv. Powder Tech. 24 (2013) 813817.

https://doi.org/10.1016/j.apt.2012.11.015

[12]H. M. Yehia, A. Abu-Oqail, M. AElmaghraby, O. A. Elkady, Microstructure, hardness, and tribology properties of the $\left(\mathrm{Cu} / \mathrm{MoS}_{2}\right) /$ graphene nanocomposite via the electroless deposition and powder metallurgy technique, J. of composite materials, 54 (2020) 3435- 3446. https://doi.org/10.1177/0021998320916528

[13]E. F EL-kashif, S. A Esmail , O. A. Elkady, B.S. Azzam and A. A Khattab, Influence of carbon nanotubes on the properties of friction composite materials, J. of Composite Materials 54 (2020) 2101-2111. https://10.1177/0021998319891772

[14]M. Hossam, F. Yehia, O. Nouh, O. El-Kady, Effect of graphene nano-sheets content and sintering time on the microstructure, coefficient of thermal expansion, and mechanical properties of $(\mathrm{Cu} / \mathrm{WC}$ TiC-Co) nano-composites, J. of Alloys and Compds 764 (2018) 36-43 https://doi.org/10.1016/j.jallcom.2018.06.040

[15]H. Peng, L. Hu, L. Li, L. Zhang, L. Zhang, Evolution of the microstructure and mechanical properties of powder metallurgical high-speed steel S390 after heat treatment, Journal of Alloys and Compounds 740 (2018) 766-773. http://dx.doi.org/10.1016/j.jallcom.2017.12.264

[16]H. Peng, L. Hu, X. Zhang, X. Wel, L. Li and J. Zhou, Microstructural evolution, Behavior of precipitates and mechanical properties of powder metallurgical high-speed steel s390 during tempering, Metall. and Mater. Trans. A 50 (2019) $874-883$.

http://dx.doi.org/10.1007/s11661-018-5040-2

[17]L. Hao, Z. Jiang, X. Cheng, J. Zhao, D. Wei, L. Jiang, S. Luo, M. Luo, L. Ma, Effect of extreme pressure additives on the deformation behavior of oxide scale during the hot rolling of ferritic stainless steel strips, Tribol. Trans. 58 (2015) 947954.

https://doi.org/10.1080/10402004.2015.1025931

[18]C. Lee, X. Wei, J.W. Kysar, J. Hone, Measurement of the elastic properties and intrinsic strength of monolayer graphene, Science 321 (2008) 385-388. http://dx.doi.org/10.1126/science.1157996

[19]O. El-Kady, H. M.Yehia, F.Nouh, Preparation and characterization of $\mathrm{Cu} /(\mathrm{WC}-\mathrm{TiC}-\mathrm{Co}) /$ graphene nano-composites as a suitable material for heat sink by powder metallurgy method, International Journal of Refractory Metals and Hard Materials, 79 (2019) 108-114.

https://doi.org/10.1016/j.ijrmhm.2018.11.007

[20]P. Nyanor, O. ElKady, H. M. Yehia, A. S. Hamada, K. Nakamura, M. A. Hassan, Effect of Carbon Nanotube (CNT) Content on the Hardness, Wear Resistance and Thermal Expansion of InSitu Reduced Graphene Oxide(rGO) Reinforced Aluminum Matrix Composites, Metals and Materials International, 5 (2019). https://doi.org/10.1007/s12540-019-00445-6 